\title{
2006-2042: REPRESENTATION ISSUES IN ENGINEERING EDUCATION: ENGINEERING ISSUES AND PARALLELS FROM THE VISUAL \& PERFORMING ARTS
}

\section{William Lee, University of South Florida}

Bill Lee is a Professor of Chemical Engineering with a significant interest in the practical and philosophical aspects of the educational process. He currently has several projects with faculty in the Visual and Performing Arts, exploring issues in the educational process, problem solving, and creativity.

\section{Mernet Larson, University of South Florida}

Mernet Larson is a Professor of Art History who has written and taught in the areas of art history, art theory, art criticism, and educational aspects of art. She is also a professional artist.

\section{Jack Heller, University of South Florida} Jack Heller is a Professor in Music Education who has written extensively in the areas of music composition, music theory, and educational and psychological issues of music.

\section{Douglas Lunsford, University of South Florida}

Douglas Lunsford is an educational psychologist specializing in the areas of educational assessment and the psychological issues related to the general educational process. 


\title{
Representation issues in engineering education: engineering issues and parallels from the visual and performing arts
}

\begin{abstract}
Engineering educations routinely use a variety of means to assist in the education process, including all forms of media (books, visual aids, virtual sources, etc.). The incorporation of "real world" materials and experiences is disappearing. Students actually see the hardware aspects of their subject less and less, including (small scale) individual components and (large scale) industrial processes. While this may reflect economic and convenience realities, there is a potential conceptual error being made by instructors (and subsequently adopted by students) that studying models, diagrams, illustrations, etc. of engineering objects or processes of interest is equivalent to (and perhaps even superior to) including the "real" object in the educational process. In the extreme, many may even feel such inclusion or the providing of such experiences is unnecessary. Put another way, elimination of "real world" experiences is accompanied by the loss of sensory information; this loss of information lessens the quality of the educational experience. In the Visual and Performing Arts (VPA), a similar problem has also occurred. Musicians study scores rather than listen to music; theater studies the script and not the performed play, etc. Thus, meaningful parallels can be drawn between engineering and the VPA in terms of the problem and its potential solutions.

A discussion of representation theory will be presented along with potential errors that may result when "reality" is progressively eliminated from direct experience. A series of examples from current engineering practices will then be presented, drawing from several engineering disciplines. Next, parallels in the VPA will be addressed, further illustrating and expanding the discussion. Finally, solutions to the problems will be presented, providing educators with practical ideas to implement. Data from a survey of engineering educators from several departments will also be included in the presentation, illustrating the problem with data showing the extent to which "real objects" are or are not included in the educational process. This includes a "snapshot" of the present situation and a comparison to earlier time periods (specifically 10 and 20 years ago). Not surprising, there is a correlation between the value of "real object" incorporation and extent of previous industrial experience.
\end{abstract}

\section{Introduction}

The following is a passage from the 1997 movie Good Will Hunting, specifically from a scene where the psychologist (played by Robin Williams) is talking with his patient (played by Matt Damon) ${ }^{1}$ :

So, if I asked you about art, you'd probably give me the skinny on every art book ever written. Michelangelo. You know a lot about him. Life's work, ... right? But I bet you can't tell me what it smells like in the Sistine Chapel. You've never actually stood there and looked up at that beautiful ceiling. ....

I ask you about war, you'd probably uh...throw Shakespeare at me, right? "Once 
more into the breach, dear friends." But you've never been near one.

In similar fashion, educator Pognowski wrote ${ }^{2}$ :

How would we describe the ocean if we had never been tugged and drawn by its current or felt the panic of its unexpected waves? Could we understand buoyancy without the experience of being held by the water? Experience is probably the best Foundation for learning. Once we have tasted the salty seasoning of the water and felt its power, we can expand what we know through novels, encyclopedias, films, and lectures. If we have never experienced the ocean, we could still read about it, but we would not remember the information in quite the same way. Such information takes on more meaning when generated by experience.

These passages illustrate the importance of direct sensory experience as a means to obtaining a broad knowledge of the subject at hand. The Good Will Hunting passage illustrates the potential incompleteness of information gleaned from the study of representations of specific objects, specifically representations presented in books. The representation of the Sistine Chapel as a visual photograph provides an experience that is but a poor imitation of the much broader sensory experience one had when viewing the real thing. Similarly, the verbal description of an ocean as referenced in the second passage pales with the direct experience of a real ocean, especially by someone who has never experienced an ocean. Both passages suggest that representation as a means of effective communication may have inherent problems.

In a broader sense, science and technology routinely rely upon visual representations to efficiently communicate concepts and summarize information. By "representation", it is meant that some visual symbol (often simple in nature), diagram, or other communication device is employed to represent or model a more complex "real" object. Often the represented object is in fact "real". For example, a mechanical engineering text may present a simple diagram intended to represent an actual pump (see Figure One). Sometimes the represented object is an estimate of the "real". For example, chemists and physicists use various representations to present models of what is currently not directly observable in the subatomic world.

Table One presents a list of "real" objects and their representations. In many cases, a representation is necessary because the real object is not immediately or easily available to the intended audience. For example, the process flow sheet is an efficient and convenient way of visualizing the actual processing facility that is usually very large and may not be geographically available. Educational institutions may use representations (increasingly in a virtual environment) because cost and space constraints may not permit direct display of the real object. Representations as such are an increasingly common and important feature of the modern communication landscape.

Normally, there is an assumed correspondence between the representation and the represented object; representations usually refer to objects that are "real" somewhere. The reader may or may not actually have first hand experience with the "real" object. If there is no first-hand experience, the reader may or may not be able to accurately mentally "reconstruct" the original 
represented object.

The objective of this paper is to point out that while representation per se plays a very important role in science and technology, it is not without its pitfalls. This is especially important to the educational process, where representations (including the increasingly present "virtual reality") are very prominent. However, these issues are relevant to the broad practice of engineering and associated technical communications. Excessive reliance upon representation can lead to the following problems:

1) Heavy reliance upon visual imagery, at the expense of the other senses

2) Losing track of the "real" object that is being represented, which may include a limited (and possibly inaccurate) appreciation for size, weight, extent, and other physical attributes

3) Related to (2), errors in mentally reconstructing the "real" object that is being represented

4) Developing the viewpoint that direct experience of the "real" object is in fact not necessary. Stated alternately, the "virtual" world of representations and manipulation of representations is itself "reality".

While focusing on science and technology, discussion will be supplemented by similar difficulties and challenges in other fields, including the visual and performing arts.

\section{Background}

The Shorter Oxford English Dictionary provides a working definition of "representation":

1. To represent something is to describe or depict it, to call it up in the mind by description or portrayal or imagination; to place a likeness of it before us in our mind or in the senses.

2. To represent also means to symbolize, stand for, to be a specimen of, or to substitute for.

Representation therefore involves several issues: the represented or intended object, the representation device or means, and the human cognitive processing of the representation as perceived by the senses (usually vision). The topic of representation can be analyzed from a philosophical, pragmatic, and social viewpoint.

\section{Plato's viewpoints}

The ancient Greek philosopher Plato was one of the earliest thinkers to address the issue of representation. Plato's concept of the Forms was a foundational component of his philosophy of Platonic Realism. To Plato, the Forms were timeless perfect abstract qualities or properties that have a real existence independent of human awareness. All material objects were but copies or particulars of the universal Forms. Thus a red apple (being a particular) is a copy of the form of 
"appleness" and also a copy of the form of "redness". The proper goal of humans who seek true knowledge is to attempt to grasp the essence of the Forms. This leads to Plato's general feeling of contempt towards the arts, including the process of representation. To Plato, an artist who paints a picture of an apple while studying an actual apple is not doing right for the viewer. The apple painting is a copy of the particular apple which is itself a copy of the Form of appleness. Instead of directing the viewer to the Form directly, the artist is directing the viewer to a copy (in fact, a copy of a copy). Thus the visual artist, by producing representations, is not performing a noble activity.

This concept is further illustrated through Plato's Allegory of the Cave. Briefly stated, prisoners incarcerated from a very young age are chained within a deep cave in such a way that they can only view the back wall of the cave. A fire behind them casts shadows of puppets and other shapes that are manipulated by men between the prisoners and the fire; all the prisoners see are the created shadows. The prisoners come to believe that the shadows are the real objects rather than recognizing that the shadows are but copies of the "real" objects unperceived behind them; they mistake appearance for reality. When the prisoners are released, they can turn their heads and discover their error. Plato would maintain that most humans exist in the Realm of Belief, i.e., all we know are the shadows which we mistake for reality. Furthermore, many of us are content to exist in the Realm of Belief and are very reluctant to pursue the Forms, i.e., seek entry into the Realm of Knowledge.

It seems likely that Plato would argue against representation practices since such practices would direct the viewer towards a "copy" of the represented object rather than towards the "real" object.

\section{Western science's empirical roots}

Much of Western science's foundational tenets trace back to the $18^{\text {th }}$ century British Empiricists Locke, Berkeley, and Hume. To the Empiricists, knowledge acquisition starts with and critically depends on sensory experience (unlike the Rationalists such as Descartes who distrusted the senses). Thus modern scientific method is very oriented towards direct observation and experimentation. Kant took it a step further, opining that we can only know about "things" as a result of sensory experiences that are processed by the mind, in a sense resolving some of the conflict between Empiricism and Rationalism. It logically follows that knowledge acquisition increases in difficulty as direct sensory experience is mitigated.

\section{Language and signs}

Culture involves "shared meanings", "shared codes" or "shared conceptual maps"3. Language is an important medium which individuals and societies ("cultures") employ to make sense of things. "Language" is taken to be a broad concept, including both linguistic components such as the written and spoken word, and nonlinguistic components such as images, objects, arts and crafts, fashion, etc. employed by the culture that can express meaning. Hall observes ${ }^{4}$ : "In language, we use signs and symbols - whether they are sounds, written words, electronically produced images, musical notes, even objects - to stand for or represent to other people our concepts, ideas and feelings." Indeed, the study of representation by a particular culture 
provides much insight into how that culture communicates, what common values are held, and even insights into the culture's creative process.

As implied above, the term "signs" broadly includes words, sounds, objects or images that can convey meaning; "signification" is the use of signs to convey meaning. "Iconic signs" are visual signs that display a recognizable resemblance to the object to which they refer. Thus a sketch of a bridge acts as an iconic sign since the resemblance to a "real" bridge is readily appreciated. "Indexical signs" include written or spoken signs that bear no obvious relationship to the referred objects. Writing the word "pump" or saying the word "pump" bears no rational relationship to the real object that is a pump.

From a historical viewpoint, iconic signs were used as representational devices long before the appearance of forms of written language. From a technological viewpoint, such signs were the first form of information storage. Note that even the most simplistic iconic signs, when combined with other signs, can convey significant meaning in spite of relatively sparse sensory information. Cognitive psychology has studied in detail the phenomena related to the extraction of meaning from iconic signs, including the mental visualization of three dimensional objects in response to the presentation of two dimensional representations ${ }^{5}$.

\section{Representation theory}

There are three broad theoretical frameworks from which one can explore the subject of representation: the reflective, the intentional, and the constructionist theories. In the reflective approach, also known as the mimetic approach, language acts like a mirror, reflecting or imitating the world that exists "out there". It is the reality "out there" that fixes meaning. Thus the word "pump" has meaning as a result of its reflection of or correspondence to a "real" pump. Almost opposite in viewpoint, the intentional approach holds that signs mean only what the presenter (author, speaker, etc.) intends them to mean; meaning in language is fixed by the individual user. Thus "black" may refer to an emotional state as intended by the poet; there is no correspondence with a tangible reality. The constructionist approach reflects the social aspects of language. Cultures construct meaning via their shared codes of concepts and signs. The members of the culture collectively agree on the meaning of signs. Thus there is a societal agreement that a flashing yellow light indicates "caution".

The linguist Saussure developed much of social constructionist theory, focusing on the complex relationship between "out there" reality, concepts, and language, breaking down "signs" into two components, the type of representation (the "signifier") and the mental concepts or ideas (the "signified") ${ }^{4}$. Saussure's work laid the foundations for what would become known as semiotics, or the study of sign use in culture. Whereas linguistics is the more focused study of language (written and spoken), semiotics is the broader study of signs, representation, and the associated cultural practices.

Many scientists have tended to agree with the reflective approach, since this realist viewpoint conveys a certain ontological status to the represented object. More specifically, this realist viewpoint assumes a real objective reality "out there", a foundational assumption of contemporary empirical science. However, it is not necessary to think of reflective/realist and 
socially constructive ("anti-realist") theories as mutually exclusive, for the technical "culture" must in fact agree to and subsequently share their codes as to the correlation or mapping between the represented object and the representation device or sign. In fact, much of the scientific representation literature draws from both viewpoints ${ }^{6}$.

\section{The visual arts}

Like their scientific counterparts, many visual artists also espouse a realist-based viewpoint. Socalled representational art is just that: art that represents "real" objects. This was the dominant approach to visual art until relatively recent times. The later $19^{\text {th }}$ century Impressionists launched the modern movement into nonrepresentational art, where visual art works often had no correspondence with "real" objects. Thus visual art, at least in the views of some, lost its way when the realist viewpoint was abandoned in nonrepresentational art. This detachment to reality would lead some art critics/historians to declare that "art is dead"7. There has been something of a backlash within segments of the art community in the latter half of the twentieth century, advocating a return to more representational art, i.e, art with some correlation to reality ${ }^{8}$.

Similar to the constructivist stress on the role of cultures, many artists also recognize an interactive role between their "representations" and the culture within which they and their viewers exist. Taylor observes": "Works of art do not spring into being as isolated phenomena but are created as part of normal human activity, reflecting the judgements, the taste, the human evaluation of the artist who conceived them and often of the time of which they are a part." Similarly, Harrison writes ${ }^{10}$ : "Interpreting art requires an understanding of the relationship between the artwork and the prevailing 'culture'."

\section{“Anti-realist” viewpoints}

Perhaps the extreme of the "antirealist" movement in general modern culture relates to what the French theorist Jean Baudrillard describes as the "simulacrum" "11. In this postmodern viewpoint, the "real" is created by the model or the simulation that has no origin in reality. In the realist viewpoint, models or simulations seek to capture characteristics of the "out there" reality, i.e., the external reality came first, then the model or sign. In the simulacrum viewpoint, the model or simulation (sign) comes first, which then produces a "reality" as a result, or, in a sense, a world of hyperreality. Thus signs no longer have any reference to "the real"; the difference between the representation and "reality" becomes very blurred ${ }^{12}$. Baudrillard alternately describes a simulacrum as a copy of a copy that is so dissipated that the original "reality" is no longer discernable.

As one popular example of simulacra, advertising (coming first) eventually creates a hyperreal world of idealistic persons living in highly idealized environments. Advertising did not seek to mirror real society and real people; rather, advertizing creates its own "reality". Fictional/fantasy movies routinely create hyperrealities (for example, the recent Matrix movie series). Baudrillard writes ${ }^{11}$ : "Today's abstraction is no longer that of the map, the double, the mirror, or the concept. Simulation is no longer that of a territory, a referential being, or a substance. It is the generation by models of a real without origin or reality, a hyperreal. The territory no longer precedes the map, nor does it survive it ... Something has disappeared ... the 
magic of the concept and the charm of the real. This imaginary of representation ... no more mirror of being and appearances, of the real and its concept."

\section{Current trends in the capstone design class: a survey}

Twenty-six programs in either chemical (10) or mechanical (16) engineering responded to a survey conducted in the spring/summer of 2005 that probed the extent to which representation is used versus direct exposure to real objects in the senior capstone design course. Twelve programs had a two semester design sequence; the remainder (14) had a single capstone course. Respondents were informed that their identification would be kept anonymous. Table 2 summarizes the results. For the "20 years ago" assessments, the responders were referencing their personal observations 20 years previously or at least their understanding of the conditions 20 years previously in terms of their present institution.

The results clearly indicate a movement away from "hands on" experience or "direct observation" of real objects/systems. Interestingly, many instructors reported that the activities currently conducted within a virtual environment were doing an adequate job of providing "design" experience. Also curious, many instructors felt that "hands on" experiences were not a particularly important component of the design course. It is speculative as to the extent that this movement away from hands-on experiences and incorporation of real world experiences reflects the growing trend in engineering education where a significant fraction of the faculty have not actually worked in a non-academic "real world" environment.

It should be noted that the survey did not probe similar issues in other undergraduate courses. The senior capstone design course was selected as the single course type for the survey in that it stressed design/analysis/synthesis on a larger scale rather than emphasizing single units or components. Also, following a series of fundamental classes, such a course would be expected to be using the developed discipline representation vocabulary extensively.

\section{Discussion}

Contemporary engineering education employs an extensive vocabulary of signs and symbols to represent both individual objects and more complex assemblies of objects. From a semiotic viewpoint, such signs are readily interpreted by a knowledgeable (or educated) member of engineering culture, a culture that established and collectively agreed to the deciphering code or map. Often these signs may be iconic in that there is some resemblance between the represented object and the real object. However, this claim of iconic may sometimes be difficult to support. For example, the pump picture (extreme right) in Figure 1 has a clear resemblance to the real pump. As one moves to the left through more simplified representations, the resemblance becomes progressively unclear to the point that the circle enclosing a "P" might be more accurately described as an indexical sign rather than an iconic sign.

In developing such representations, there is usually an underlying assumption that the reader/viewer of the signs actually has an accurate concept of the intended real object. Individuals who have actually worked with a real pump know very accurately what is represented by the representational objects in Figure 1. However, individuals who have never 
worked with a real pump may not have such knowledge. In fact, the individual may mentally be reconstructing inaccurate "real" pumps when processing the representational objects. Also, the "real" pump experience involves real noises, smells, tactile properties (e.g., hardness), etc. associated with real pumps. Indeed, the individual never having direct experiences of real pumps would most likely extrapolate or construct a mental model based solely on vision; other sensory attributes would most likely be minimized or totally omitted. Similar examples are readily identified in other engineering disciplines. Civil engineering students study truss diagrams but never see the actual three-dimensional system. Chemical engineering students never directly experience a real distillation column or chemical reactor. Electrical engineering students have no direct experience of the actual power plant.
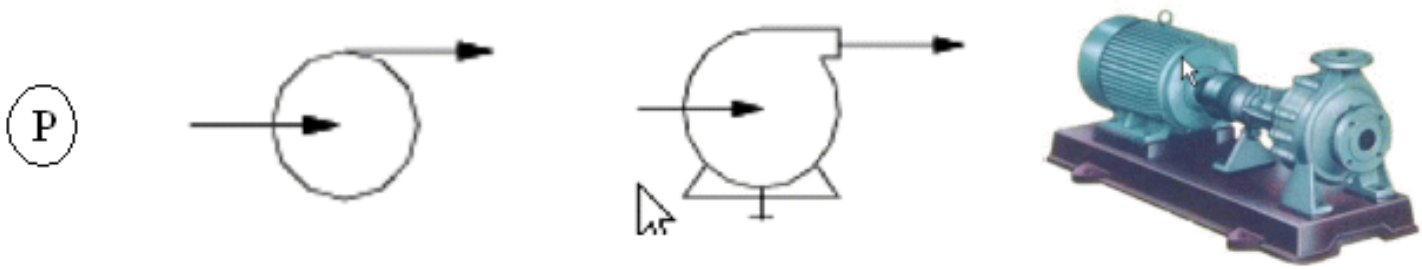

Figure 1: Possible representations of a pump.

The more indexical the sign, the more mysterious the symbol system will be to those encountering it for the first time. To someone just starting the technology educational process, a wiring diagram of a computer mother board will be very cryptic. Mentally constructing the actual hardware from the given diagram may be very challenging. Nonmusicians find music scores to be mostly meaningless; choreography sequences are perplexing to the nondancer. Regenerating the live music or dance performance with any accuracy is unlikely. It is only through an educational process involving "real" experiences that the sign and symbol system can be deciphered to the point of being meaningful.

The current technology of representation goes beyond the culturally developed system of signs. More engineering students work in a virtual environment, with this trend likely to continue to the point of becoming the dominant presentation means. Students arrange/rearrange signs as they model circuits, processing schemes, building structures, biological processes, etc. As this process proceeds without any "grounding" or calibration with the real objects, students may lose track of the reality they may claim to be modeling, studying, designing, etc. In the worse case, subsequent attempts at reconstructions of the "real" may be extremely inaccurate.

Other observations from contemporary education: Geography students study the map rather than the actual land. Musicians study the score rather than listening to the actual music. Theater majors and literature students study the script of the play rather than seeing the actual performance. Medical students study anatomical drawings, perhaps in lieu of direct cadaveric investigation. Architectural students study diagrams and photographs rather than the actual structures. Many disciplines have their particular dependence on representations in lieu of direct 
experience of the actual object. In engineering, as in these other disciplines, this is often a very efficient way of conducting design, analysis, and synthesis activities. Symbolic manipulation may be a good initial environment for later more efficient real object manipulation. Exploring "what if"s?" by symbol manipulation and model testing can make the later experimental phase more focused. For example, engineering students have many more degrees of freedom to manipulate the object of interest and explore various outcomes very easily in a virtual environment, often before returning to the "real" physical system. In their version of a virtual environment, musicians can easily explore instrument changes and significant score alterations before transitioning to the live performance. "Closing the loop" education should at least periodically have access to the "real" object; this provides calibration and significance to the representational system.

Such representational or signification processes are often exclusively aimed at the sense of vision. Thus the movement to representational signs is often synonymous with the removal or denying of non-vision sensory experiences. Our signs and symbols usually produce visual stimuli, not acoustic, tactile, taste, or smell stimuli. In the spirit of the Good Will Hunting quote, imagine studying a diagram of a guitar and proceeding to claim detailed knowledge of a "guitar" without ever having played or listened to one. Imagine studying a recipe for a particular dish and then claiming to have an appreciation for the culinary aspects without ever experiencing the actual eating experience. Without a basis of direct knowledge of the real or represented object(s), one might perhaps be able to reconstruct or synthesize a reasonable visual image of the real object, but would not be able to reconstruct or synthesize the other sensory experiences that would also accompany the real object in a real environment.

Two other potential errors must be considered. First, there is the error where one feels that it is no longer necessary to experience real objects, to "calibrate" one's representational vocabulary. The virtual designer may no longer see a need to venture out from behind the computer screen. This has been an increasingly recognized problem in the human factors area. Display-based training systems (for example, flight training virtual systems) are often increasingly realistic; however, problems in spacial and temporal perception result when no actual "real" experience supplements the virtual-based training ${ }^{13,14}$. Second, and perhaps more serious, is when one (in the absence of direct experience of the real) proceeds to reconstruct reality from the representational system, in effect creating a hyperreal or simulacrum. Thus the constructed "reality" may not map at all into the actual "reality". Theoretical physics sometimes encounters this problem, reflecting the difficulty that the subatomic particles of interest simply cannot be visualized directly ${ }^{15}$. Also, engineering students who are never exposed to the real objects during their education will nonetheless (in Kantian fashion) proceed to fill in the gaps and otherwise construct their own version of reality. The probability of constructing incorrect "realities" would be significant.

Others have observed that the current generation of secondary education students (and many college students) are very hands-on learners as indicated by their extensive use of computer and video games. College instructors should take advantage of this already-developed learning process by presenting hands-on learning experiences ${ }^{16}$. In secondary science education, it is often stressed that effective science teaching should involve "hands on" multisensory experiences ${ }^{17,18}$. Indeed, the so-called "Montessory Method" is based on extensive multisensory 
experiences as a prerequisite for effective learning, particularly at an early age ${ }^{19}$ (Montessori, 2002). Such an approach is consistent with Gardner's theory of multiple intelligences that argues that there are many forms of intelligences that can be mastered by an individual. It should be noted that a passive visual-based presentation does not address "multiple intelligences" to the extent that hands-on multisensory active approaches accomplish ${ }^{20}$.

\section{Recommendations}

The educational experience should incorporate student "reality" experiences as much as possible. Students should see, hear, touch, etc. the actual objects that are the focus of their chosen discipline. Minimization or complete absence of hands on activities or direct multisensory experiences can be very problematic. Reality exposures will allow students to periodically calibrate their representational vocabulary, allowing them a more accurate understanding of the real objects that are intended by the engineering design and analysis process. Engineering is a pragmatic enterprise intended to provide useful "objects" to society; an inability to successfully appreciate what real objects are really like, or, inaccurately developing a hyper-reality that simply does not map into the real world is counterproductive; at least, it is inefficient.

Educational experiences outside the classroom, away from the computer screen, beyond the textbooks and websites, should be provided as possible; such experiences should not be minimized or considered nonproductive. Music majors should experience the live performance; artists should visit museums to view artwork directly; theater majors should experience the live play. For the engineering students, visit the manufacturing facility, power or chemical plant, built structure, landscaped property, etc. Let the students fully experience in a very direct fashion the intended outcome of their labors. Stimulate their eyes AND their other senses. Coop programs were designed (and often encouraged) with the idea that students get to experience reality during their education. As such, co-op or intern programs serve a very important role in providing calibrations for the discipline's developing representational system.

From Plato's viewpoint, educators should strive to focus (or remind) students of the "out there" object of which the representation is just a copy or reflection, striving to keep students grounded in the "realm of knowledge". Employing educational approaches that rely on "copies" or "copies of copies" always run the risk of losing track of the "real" original, that which should be the ultimate object of one's learning. In the spirit of our empirical tradition, fully employing all our senses is the pathway to knowledge; reducing the sensory experience to a visual symbol with no subsequent or parallel experience of the represented reality can only lead to incomplete (and possibly incorrect) knowledge.

\section{Acknowledgements}

This work was supported by NSF grant number 0235214. 


\section{References}

1. Affleck, B. and Damon, M. Good Will Hunting. Gus Van Sant (director). Walt Disney Video, 1997.

2. Pogonowski, L. Developing skills in critical thinking and problem solving. Music Educators Journal 73(6): 37-41. 1987.

3. Du Gay, P., Hall, S., Janes, L, Mackay, H, and Negus, K. Doing cultural studies: The case of the Sony Walkman. The Open University, 1997.

4. Hall, S. ed. Representation: Cultural representations and signifying practices SAGE Publications, 2003.

5. Massironik, M. The psychology of graphical images: Seeing, drawing, communicating Lawrence Erlbaum Associates Publishers, 2002.

6. Tibbetts, P. Representation and the realist-constructivist controversy. In: Representation in scientific practice M. Lynch and S. Woolgar, eds., The MIT Press, 1988.

7. Danto, A. The philosophical disenfranchisement of art. Columbia University Press, 1986.

8. $\quad$ Foster, H. The return of the real The MIT Press, 1996.

9. Taylor, J.C. Learning to look: A handbook for the visual arts University of Chicago Press, 1981.

10. Harrison, C. Essays on art and language The MIT Press, 2001.

11. Baudrillard, J. Simulacra and simulation The University of Michigan Press, 1994.

12. Reed, C. Postmodernism and the art of identity. In: Concepts of Modern Art: From Fauvism to Postmodernism N. Stangos, ed. Thames \& Hudson, 1994.

13. Smallman, H.S. and St. John, M. Naive realism: Misplaced faith in realistic displays. Ergonomics in Design 13(3): 6-13, 2005.

14. Hamilton, R.M. and Holmquist, J.P. Training in virtual and augmented realities: An interview with Bruce Knerr. Ergonomics in Design 13(3): 18-22. 2005.

15. Schlain, L. Art and physics: Parallel vision s in space, time \& light Perennial, 1991. (of particular interest, see Chapter 17 "Abstract art/Imageless Physics").

16. Wankat, P. and Oreovicz, F. Gaming the curriculum. ASEE Prism 15(1): 48, 2005.

17. Pourde, L.A. and Klemm, E.B. Sounds and sense-abilities. College Student Journal 38(4): 653-660, 2004.

18. Berk, E.G. Hands-on science: using manipulatives in the classroom. Principal 78(4): 52, 1999.

19. Montessori, M. The Montessori method Dover Publications, 2002.

20. Vardin, P.A. 2003. Montessori and Gardner's theory of multiple intelligences. Montessori Life 15(1): 4043. 


\section{Table One}

\section{Representation examples}

\section{$\underline{\text { Representation }}$}

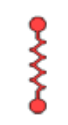<smiles>[AlH2]</smiles><smiles>c1ccccc1</smiles>

map

process flow diagram

circuit diagram

anatomical drawing

house or building plan

neural net model

picture or painting of a flower vase

script

score

\section{"Real" object represented}

resistor

valve

benzene molecule

actual country or region

existing (or proposed) processing facility

actual circuit device

living human body

existing (or proposed) house or building

"real" human learning

the actual flower vase

the performed play

the performed music 


\section{Table Two}

Summary of the survey results of chemical (ChE) or mechanical (ME) engineering senior capstone design course

Responding programs

Senior design currently conducted within a mostly virtual environment

Extent to which this was true 20 years ago
$26(10 \mathrm{ChE}, 16 \mathrm{ME})$

ChE: 10

ME: 14

ChE: 4

ME: 2
Students worked with real hardware, visited an actual plant, etc. during course

Extent to which this was true 20 years ago
ChE: 2

ME: 6

ChE: 10

ME: 16

If there is a difference, why?

"Virtual" experience is better Cost limitations/cannot afford hardware/ limited in-department infrastructure No strong justification for such activities Takes too much time/scheduling challenges Limited availability/access (off campus) Students are not interested in non-virtual experiences

Extent to which the main instructor(s) had industrial experience

ChE: 4

ME: 5

Extent to which this was true 20 years ago

ChE: 9

ME: 12 